\title{
The effect of acupuncture and static stretch on the flexibility of the hamstring muscles
}

Volume 5 Issue 4 - 2017

\section{Introduction}

Muscle flexibility, as defined by Zachezeweski, " "is the ability of a muscle to lengthen, allowing one joint (or more than one joint in a series) to move through a range of motion ROM" and a loss of muscle flexibility as "a decrease in the ability of the muscle to deform," resulting in decreased ROM about a joint. Much literature has been written on the importance of flexibility in normal muscle function, the prevention of injury, and the enhancement of sports performance. ${ }^{2}$ Good flexibility will allow muscle tissue to adapt to imposed stress more easily and allow efficient and effective movement. ${ }^{3}$

In the rehabilitation, physical therapy, and sports medicine settings, many therapists use stretching exercises to improve ROM and function after trauma and periods of immobilization. Better ROM will help prevent injury, and reduce muscle soreness after physical activity.

There are many studies that show that the muscle stiffness curve does not change after a 2-4-week stretching program or after 10 minutes of sport stretching. ${ }^{3,5,6}$ It has been hypothesized that muscle stiffness would diminish and become more flexible if acupuncture therapy was added to the stretching program within this 2-week period. Reducing therapy frequency and achieving quicker more effective results is of great health and economic value.

The purpose of this pilot study is to create a quick and effective, yet safe, way of increasing muscle flexibility with the use of acupuncture and static stretching, as opposed to a static stretching routine alone. Increased ROM of tight hamstring muscles will be attempted within a 2-week period.

Methods of stretching include dynamic stretching, ballistic stretching, static stretching, and variations of Proprioceptive neuromuscular facilitation techniques. Although documentation exists that all forms of stretching will increase the flexibility of muscle, we believe that the most common and safest method is the static stretch. ${ }^{2}$

Short, tight muscles that decrease ROM predispose the body to disease, injury, and delayed healing. Although the mechanism(s) of Acupuncture is unclear, it is hypothesized that intramuscular needle stimulation for releasing shortened muscle will decrease pain (or increase pain tolerance) and improve joint range. Treating the muscle with electric needle stimulation may also increase the relaxation of the entire muscle.

To date, no objective study has examined the effects of acupuncture therapy with the use of static stretching to better increase flexibility of the hamstring muscle. In addition, no research has been conducted to show that acupuncture will decrease the frequency of a static stretch routine necessary to achieve efficient results.

\section{Methods}

\section{Subjects}

There existed 4 criteria for participation in the study. First, each

\author{
Christopher M Chapleau \\ The Connecticut Society of Acupuncture \& Oriental Medicine, \\ USA \\ Correspondence: Christopher M. Chapleau, The Connecticut \\ Society of Acupuncture \& Oriental Medicine, USA, Tel 203-56I- \\ 3276, Email cchapleau555@gmail.com
}

Received: October 26, 2016 | Published: February 16, 2017

subject agreed to volunteer and complete the 2 weeks of training. Second, the subjects could not have any recent or chronic pathology to the hip, knee, thigh, or lower back. Third, each subject had to exhibit tight hamstring muscles, operationally defined as having greater than 15 loss of knee extension measured with the femur held at 90 of hip flexion. Finally, subjects not involved in any exercise activity at the start of the study had to agree to avoid lower extremity exercise, and those already involved in a regular exercise program agreed not to increase exercise intensity or frequency throughout the 2 weeks. ${ }^{4}$

6 subjects ( 4 men and 3 women) with the mean age of 27.33years met the established criteria and completed the study. All subjects signed an institutional approved informed consent statement prior to data collection.

\section{Equipment}

Measurements were performed using a double-armed, full circle protractor (True Angle Goniometer, Novel Products, Inc.) made of transparent plastic. The protractor measured degrees in 1 increment. To ensure appropriate reliability, the goniometer was modified by taping 12 inch wooden ruler extensions to its arms. This increased the length of the arms to $43 \mathrm{~cm}$ (17inches). The rationale for adding the extensions was that, in doing so, the distance between the protractor arm and the marked bony landmarks was decreased. The extensions allowed for the preservation of accurate measurement of hamstring flexibility while decreasing measurement time (2\&4).

\section{Procedure}

Prior to assignment to group, each subject who met the criteria for inclusion in the study was measured for flexibility of the right hamstring muscle (arbitrarily chosen). Subjects were positioned supine with the right hip and knee flexed to 90 . In this position, the leg landmarks were marked with a felt tip pen. The Landmarks were: the greater trochanter of the femur, the lateral malleolus and the lateral epicondyle of the femur. During measurement, one researcher 
positioned the right hip in 90degrees of hip flexion. A second researcher passively moved the tibia to terminal position of knee extension. This was defined as the point at which the subject complained of a feeling of discomfort or to the point that the researcher perceived resistance to the stretch. Once the terminal position of the knee extension was reached, the first researcher measured the amount of knee extension with the goniometer. Zero degrees were considered to be full knee extension. No warm-up prior to data collection was allowed. ${ }^{2}$ The same examiner made all the measurements for the entire study.

Subjects were randomly assigned to one of two groups following the initial measurement of hamstring flexibility. Subjects assigned to Group $1(\mathrm{~N}=2$ men, 1 women; $\mathrm{X}$ age $=33)$ were given 1 acupuncture treatment and stretched five times a week for 2 weeks. Group 1 had a total of 2 acupuncture treatments each spaced seven days apart. Group 1 performed static hamstring stretches by standing erect with the left foot on the floor and pointing straight ahead with no rotation in the hip. The right hamstring muscles were stretched by placing the right heal on an elevated surface with the knee fully extended, toes pointed to the ceiling, no rotation of the hip, and arms flexed to shoulder level. The elevated surface was high enough to cause a gentle stretch sensation in the posterior thigh. The subject then flexed forward from the hip, maintaining the spine in neutral position (back straight), while reaching the arms forward until a gentle stretch was felt. ${ }^{2,4}$ Once this position was achieved, the stretch was held for 30seconds. This was repeated 3 times with 15 seconds of rest between each stretch. This stretch was performed on their own 5 times during the week for two weeks.

Group $2(\mathrm{~N}=2$ men, 1 women; $\mathrm{X}$ age $=31.6)$ performed the same static stretch protocol 5 days a week for 2 weeks. They did not receive acupuncture treatments. Each subject was given a sheet of paper with the stretch protocol and also given a demonstration of the stretch. After 2 weeks, all subjects were retested using the same procedures described in the initial testing.

\section{Data analysis}

Values are given as Mean + SEM ( $\mathrm{n}=3$ ). Data were analyzed by unpaired Student's t-test and one-way ANOVA followed by Tukey's post hoc test. Values between the two groups differed significantly at $* \mathrm{p}<0.001$

\section{Conclusion}

Based on the results, motor point acupuncture with a static stretch routine performed every week for 2 weeks shows a statistically significant difference in hamstring ROM between the two groups at $\mathrm{p}<0.001$.

This pilot study was an initial experiment into a study I feel could be extremely beneficial to the health of many. Time and money are strong determinants in the character of a study. However, considering these obvious limitations as a student, the following are also considerations into future studies of this nature:

i. This study was limited to the effects of treating the hamstring muscle.

ii. Other acupuncture techniques, procedures/protocols and points may be more effective. Such as: use of moxibustion; needling the antagonist muscle group, auricular acupuncture, etc.

iii. Increase acupuncture treatment frequency.

iv. Type of stretch and treatment frequency per week may be changed to be more effective. v. Supervise and observe stretch routine to ensure that it is performed correctly.

vi. Increase study duration to 4 weeks.

vii. Have a "sham" acupuncture group to "blind" the subjects.

viii. "Blind" the examiner taking the pretest and posttest measurements. The examiner would be unaware of what group each subject is in.

ix. Use a much larger population.

x. Age can be a factor.

\section{The acupuncture protocol from a western medical perspective}

In Traditional Chinese Medicine (TCM), needling a patient and giving them a balancing treatment, often helps alleviate the pain. However, in many cases, the pain may come back due to a muscle imbalance problem that offsets the body's biomechanical alignment. The Musculoskeletal system is like a pulley and lever system in which the key to structural balance is in an equal pull by the opposing muscle groups located on each side of the joint. An imbalance between the forces, from an adaptive shortening on the one side, to a stretch weakness on the other, usually results in a difference of range of motion. The discordance between the antagonist and agonist muscle groups predisposes the musculoskeletal system to injury and soreness. ${ }^{7}$

The zone of muscle innervations is normally found centrally along the muscle fibers where it has the best advantage for mechanical efficiency to affect the entire muscle by the innervating nerve fibers.? Therefore, the most effective sites for needle insertion to release short, tight bands of muscle are deep to the motor point at the muscle zone of innervations where the most electrical influence of the entire muscle can be obtained. ${ }^{7-10}$

Acupuncture to the motor points seems to "reset" the dysfunctioning muscle spindle that is causing abnormal muscle function and reflexive spasm (that may be elicited by the needle). The acupuncture needle is one of the best physical therapy modalities to use, as it releases muscle shortening quickly and accurately when inserted into a motor point. $^{7}$

Points located 5 to $1 \mathrm{inch}$ along either side of the spine between spinous processes of lumbar 4 to sacral foreman 2 where needled. The Chinese name for these points are called Huatuo Jia Ji points. The concept behind needling these points from a western perspective is that they will stimulate the particular spinal nerve roots that innervate the hamstring muscle regions. The idea is to facilitate these segments and viserosomatic reflexes that affect pathological change of the spinal nerve and all the tissues supplied at that level. The paraspinal muscles that support this area between L-4 and S-2 may be short and tight creating pressure on the disk and nerve root. These muscles pull the adjacent vertebrae together which impedes the nerve impulse, thus creating muscle imbalance. ${ }^{7-10}$

\section{Motor Point Locations: Biceps femoris-3 motor points}

a. Short head- 5cun directly superior from UB 38.

b. Long head -

A. 1cun lateral from UB 37.

B. 1-1.5cun inferior and slightly lateral from UB 37. 


\section{Semitendinosus- 2 motor points}

a. 2cun medial and superior from UB 37

b. 2cun medial and 1cun inferior from UB 37

\section{Semi membranous- I motor point}

a. 6cun superior from $\mathrm{K} 10$

b. Motor Points $=$ Black Xs

c. UB Points $=$ Red dots

$*$ Cun $=$ Chinese term for an anatomical measurement unit which is proportional to each individual (1cun is approximately 1 inch $+/$ $1 / 3$ inch).

\section{Hamstring muscle static stretch protocol}

Standing hamstring stretch: Stand erect with the left foot on the floor and pointing straight ahead with no rotation in the hip. Next place the heal of your right foot on an elevated surface with the knee fully extended, toes pointed to the ceiling, no rotation of the hip, and arms flexed to shoulder level. The elevated surface should be high enough to cause a gentle stretch sensation in the posterior thigh. Now flex forward from the hip and maintain the spine in neutral position (back straight). Gradually increase the intensity of the stretch, by leaning forward more, until discomfort (not pain) in the hamstrings is felt. Hold this position for 30 seconds. Repeat 3 times with 15 seconds of rest between each stretch. Do this stretch 5 times during the week for two weeks.

Make sure that your muscles are warm. Do this stretch inside a warm house or after you've taken a warm shower. Please do not exercise or do any extra stretching during the next 3 weeks. If you do exercise please do not change your routine (workout frequency, duration, intensity, or exercise mode). Specially avoid lower extremity exercises.

\section{Summary}

The purpose of this controlled, randomized pilot study is to create a quick and effective, yet safe, way of increasing muscle flexibility with the use of motor point (MP) acupuncture and static stretching, as opposed to a static stretching routine alone. Increased ROM of tight hamstring muscles will be attempted within a 2-week period. 6 subjects ( 4 men and 3 women) with the mean age of 28.20years met the established criteria and completed the study. Subjects were randomly assigned to one of 2 groups.

Subjects assigned to Group1 were given 1 MP acupuncture treatments and performed a static stretch five times a week for 2 weeks. Group 2 performed the static stretch routine 5 days a week for 2 weeks. They did not receive acupuncture treatments. Means and standard deviations were calculated for the pretest and posttest measurements for each group, as well as the mean difference between pretest and posttest scores, for the dependent variable knee extension ROM in degrees. Based on the results, motor point acupuncture with a static stretch routine performed every week for 2 weeks does statistically show a significant difference in hamstring ROM between the two groups at $\mathrm{p}<0.001$.

The Effects of Motor Point Acupuncture and Static Stretch on the Flexibility of the Hamstring Muscles By Christopher M. Chapleau.

\section{Acknowledgments}

None.

\section{Conflicts of interest}

Author declares there are no conflicts of interest.

\section{Funding}

None.

\section{References}

1. Zachezeweski .Improving flexibility. Physical Therapy. Philadelphia, USA: JB Lippincott Co; 1989.

2. Bandy WD, Irion JM, Briggler M. The effect of time and frequency of static stretching on flexibility of the hamstring muscles. 1997;77(10):1090-1096.

3. Bandy WD, Irion JM, Briggler M. The effect of static stretch and dynamic range of motion training on the flexibility of the hamstring muscles. JOSPT. 1998;27(4):295-300.

4. Halbertsma JP, Mulder I, Göeken LN, et al. Repeated Passive Stretching: Acute Effect on the Passive Muscle Moment and Extensibility of Short Hamstrings. Arch Phys Med Rehabil. 1999;80(4):407-414.

5. Halbertsma JP, Göeken LN. Stretching exercises: effect on passive extensibility and stiffness in short hamstrings of healthy subjects. Arch Phys Med Rehabil. 1994;75(9):976-981.

6. Halbertsma JP, van Bolhuis AI, Göeken LN. Sport stretching: effect on passive muscle stiffness of short hamstrings." Arch Phys Med Rehabil. 1996;77(7):688-692.

7. Callison. Motor Point Manual. USA: Raymert Press, Inc, California; 2000

8. Jaeger, Skootsky .Double blind, controlled study of different myofasial trigger point injection techniques." Abstract. Pain. 1987;(Suppl 4):S292.

9. O'Connor, Bensky .Acupuncture: A Comprehensive Text. Shanghai College of Traditional Medicine. Seattle: Eastland Press, Washington. 1981.

10. Liao, Lee .Principles and Practice of Contemporary Acupuncture. New York, USA: M. Decker; 1994. 